\title{
A enfermagem frente a reintegração social de pacientes com distúrbios psíquicos
}

Nursing in front of social reintegration of patients with psychic disorders

Enfermería frente a la reintegración social de pacientes con trastornos psíquicos

Theodora Maria de Paiva dos Santos ${ }^{1 *}$, Nathalia Olímpio Santos ${ }^{2}$, Patrícia Fonseca Paixão², Edméa Maria de Paiva dos Santos ${ }^{3}$, Daniella Souza Gama Siqueira ${ }^{3}$, Breno de Souza Mota ${ }^{4}$, Nataly Danielle Araújo Queiroz ${ }^{5}$, Silvana Nunes Figueiredo ${ }^{6}$.

\section{RESUMO}

Objetivo: Descrever a participação da equipe de enfermagem frente a reintegração social de pacientes com distúrbios psíquicos. Métodos: Trata-se de uma Revisão Integrativa da Literatura (RIL), onde foram utilizadas as bases de dados LILACS e BDENF. Foram inseridos os estudos publicados nos últimos cinco anos, em português, disponíveis na íntegra em texto complete e que possuia relação com a temática abordada. Resultados: Após a aplicação dos filtros, o total de artigos encontrados foi 47 e selecionados 16. Dentre as modalidades de atendimento extra hospitalar onde foi possível constatar a presença da enfermagem com maior relevância, destaca-se o serviço de residência terapêutica e CAPS. Considerações finais: $A$ assistência de enfermagem nas unidades de atendimento extra-hospitalar constitui não somente a administração de medicamentos e consultas regulares, no processo de reintegração social, realize atividades lúdicas que induzem a autonomia do indivíduo respeitando suas características singulares, investindo em propostas terapêuticas descentralizadas de acesso integral e equânime para adesão ao tratamento proposto e recuperação da saúde.

Palavras-chave: Saúde mental, Psiquiatria, Enfermagem.

\begin{abstract}
Objective: To describe the participation of the nursing team in the social reintegration of patients with mental disorders. Methods: This is an integrative literature review (ILR), using the LILACS and BDENF databases. Studies published in the last five years, in Portuguese, available in full text and related to the theme were included. Results: After applying the filters, the total of articles found was 47, and 16 were selected. Among the extra-hospital care modalities where it was possible to verify the presence of nursing with greater relevance, the therapeutic residence service and CAPS stand out. Final considerations: Nursing care in outof-hospital care units constitutes not only the administration of medication and regular consultations, but also the process of social reintegration, carrying out playful activities that induce the autonomy of the individual respecting his or her unique characteristics, investing in decentralized therapeutic proposals of full and equal access for adherence to the proposed treatment and health recovery.
\end{abstract}

Keywords: Mental health, Psychiatry, Nursing.

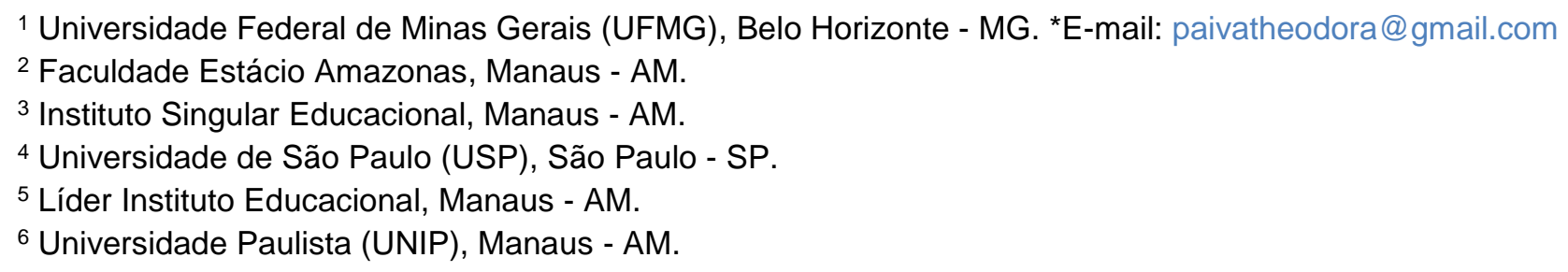




\section{RESUMEN}

Objetivo: Describir la participación del equipo de enfermería en la reinserción social de pacientes con trastornos mentales. Métodos: Se trata de una revisión bibliográfica integradora (RBI), que utilizó las bases de datos LILACS y BDENF. Se incluyeron estudios publicados en los últimos cinco años, en portugués, disponibles en su totalidad en texto completo y que estuvieran relacionados con el tema abordado. Resultados: Tras la aplicación de los filtros, el total de artículos encontrados fue de 47 y se seleccionaron 16. Entre las modalidades de atención extrahospitalaria en las que fue posible constatar la presencia de la enfermedad con mayor relevancia, destaca el servicio de residencia terapéutica y el CAPS. Consideraciones finales: Los cuidados de enfermería en las unidades de atención extrahospitalaria constituyen no sólo la administración de medicamentos y consultas periódicas, sino también el proceso de reinserción social, realizar actividades lúdicas que induzcan la autonomía del individuo respetando sus características únicas, invirtiendo en propuestas terapéuticas descentralizadas de acceso pleno e igualitario para la adherencia al tratamiento propuesto y la recuperación de la salud.

Palabras clave: Salud mental, Psiquiatría, Enfermería.

\section{INTRODUÇÃO}

O processo de contextualização dos transtornos mentais e compreensão das intervenções do Estado como política pública de saúde é uma trajetória histórica que consiste em avanços fortemente relacionados a movimentos sociais, visto que a saúde mental antes do século XIX não possuía definição própria, sendo assim, não havia distinção entre razão e loucura (CABRAL SB e DAROSCI M, 2019).

Atualmente, entende-se que os transtornos mentais possuem diferentes manifestações, são caracterizados por comportamentos anormais como emoções e percepções que comprometem o relacionamento interpessoal. Dentre esses transtornos psiquiátricos estão inclusos a depressão, esquizofrenia, transtorno afetivo bipolar, demência e autismo. Para tratamento destes distúrbios, a Organização Mundial de Saúde (OMS) descreve a importância do acesso aos cuidados de saúde com apoio social (BRASIL, 2018).

Entretanto, até meados da década de 70, no Brasil as pessoas com transtornos mentais ou déficits cognitivos eram classificadas como alienadas e diagnosticadas com moléstia mental. Em casos de importunação pública ou risco de violência, esses usuários eram recolhidos da sociedade e mantidos em um local segundo a Lei 1.132, primeira lei relacionada a saúde mental no Brasil que diz: "o indivíduo que, por moléstia mental, congênita ou adquirida, comprometer a ordem pública ou a segurança das pessoas, será recolhido a um estabelecimento de alienados" (BRASIL, 1903).

A internação hospitalar passou a ser o único tratamento de assistência psiquiátrica, onde o cuidado resumia-se na administração de medicamentos psicotrópicos, contenções e eletrochoques. $O$ portador da loucura era segregado e convivia em hospitais durante toda a vida, desse modo, surgiram os estigmas de que os loucos representavam uma ameaça social (KINKER FS, 2017).

Entre as décadas de 60 e 70, a saúde mental foi conhecida como a indústria da loucura, nessa época houve incentivo financeiro a privatização de serviços públicos em saúde resultando em ofertas de leitos enaltecendo o tratamento hospitalocêntrico, dificultando a aprovação de programas com assistência psiquiátrica descentralizada (CABRAL SB e DAROSCI M, 2019)

Com o avanço dos movimentos sociais representados por sindicatos, partidos políticos e associações que exigiam melhoras no país, fica registrado com grande impacto em 1978 o Movimento dos Trabalhadores em Saúde Mental (SMTM), incluindo os pacientes submetidos a internações prolongadas, esse fato histórico ficou conhecido como a luta antimanicomial e proclama a reforma psiquiátrica no Brasil (BRASIL, 2005).

O objetivo da reforma psiquiátrica foi denunciar as condições insalubres de habitação dos manicômios e exigir alternativas para tratamento envolvendo mudanças políticas, sociais e jurídicas. Durante essas alterações, foi observado que no tratamento hospitalocêntrico, estavam inseridos na terapia manicomial 
apenas os médicos, portanto desde o ano de 1949, tornou-se obrigatória a inserção da equipe de enfermagem. Composta por enfermeiros e técnicos especializados em saúde mental, a enfermagem iniciou a sua contribuição profissional durante tratamento intra-hospitalar exclusivo, e representa uma das primeiras categorias a compor a equipe de saúde mental do Brasil (MARTINS GCS, et al., 2018).

Essas temáticas foram abordadas na $8^{a}$ Conferência Nacional de Saúde e esses direitos são assegurados na Constituição Federal na lei 8.080/90 Art. 196, onde "A saúde é um direito de todos e dever do Estado" e, após esse avanço, as políticas em saúde mental passaram a ser mais delimitadas (BRASIL, 2005).

Em 1992, como resultado da II Conferência em Saúde Mental, passa a entrar em vigor o Núcleo de Apoio Psicossocial (NAPS) e os Centros de Atenção Psicossocial (CAPS), que são multidisciplinares, atuam em centros urbanos e atendem a grupos específicos de acordo com o número populacional. Associado a esses centros, existem outras modalidades de atendimento como o hospital dia e as residências terapêuticas (BRASIL, 2004).

As residências terapêuticas entram em prática como alternativa de apoio psicossocial extra-hospitalar, desconstruindo o antigo regime hospitalocêntrico, sendo candidatos a residir nessas moradias, pessoas que não possuem vínculo familiar nem ligação direta com unidades hospitalares ou pacientes que possuem histórico de internações recorrentes. Essa modalidade de serviço especializado, visa reinserir antigos internos em manicômios no contexto social, independente do seu transtorno ou disfunção mental (BRASIL, 2019).

Atualmente, todos os níveis de atenção especializado em saúde mental no Brasil contam com a assistência da equipe de enfermagem, estes desempenham um papel primordial no atendimento intra-hospitalar e extrahospitalar. Como consequência da descentralização do atendimento psicossocial, expandiu-se as estratégias que visam devolver a autonomia e liberdade dos pacientes que viveram reclusos em manicômios durante toda a vida, entretanto, agora são utilizados os métodos da enfermagem baseada em evidências, diferente dos conhecimentos empíricos adotados antes da reforma psiquiátrica (PINHEIRO WC, et al., 2019)

As atribuições da equipe de enfermagem especializada em saúde psiquiátrica seguem de acordo com a Resolução 599/2018, do Conselho Federal de Enfermagem (COFEN), onde descreve a importância desses profissionais e regulamenta todas as atividades assistenciais que podem ser feitas pelo profissional enfermeiro, como orientar e estabelecer vínculo com os pacientes afim de promover um ambiente terapêutico (COFEN, 2018).

Alguns estudos como o de Ermerin MF e Souza M (2016) descrevem a dificuldade do processo de melhoria dos pacientes que se mantiveram em tratamento hospitalar exclusivo e relata a negligência dos profissionais especializados durante a assistência. Mediante essas evidências, esse estudo teve como objetivo descrever a contribuição da equipe de enfermagem no processo de reintegração social dos portadores de distúrbios psíquicos.

\section{MÉTODOS}

Trata-se de um estudo descritivo, do tipo Revisão Integrativa da Literatura (RIL) baseado nas cinco etapas para a sua elaboração. O objetivo da RIL consiste em sistematizar os trabalhos existentes relacionados a um tema específico e elaborar uma síntese explicativa acerca do que foi encontrado após a busca bibliográfica. Os dados analisados das publicações anteriores permitem a interação dos resultados, sendo possível realizar comparações e aprimorar conhecimentos preexistentes, desta forma, contribui cientificamente para 0 aperfeiçoamento profissional (SOUZA MT, et al., 2010).

A pesquisa foi elaborada e dividida em cinco momentos: 1 - identificação do problema; 2 - síntese dos critérios de elegibilidade e inelegibilidade dos artigos encontrados; 3 - avaliação completa dos estudos selecionados para a revisão; 4 - interpretação e análise dos manuscritos; 5 - síntese total dos estudos.

Para alcançar a proposta deste estudo, foram definidas 2 perguntadoras, sendo elas: Os CAPS e as residências terapêuticas possuem impacto direto na melhora do estado cognitivo dos antigos internos? Quais as contribuições da equipe de enfermagem nos CAPS e nas residências terapêuticas? 
Neste estudo, as bases de dados utilizadas para a revisão integrativa foram: Literatura Latino-Americana e do Caribe em Ciências da Saúde (LILACS) e Bases de Dados de Enfermagem (BDENF). Para a busca bibliográfica, foram selecionados os descritores em ciências da saúde (DeCS), Saúde mental, Psiquiatria e Enfermagem, utilizando o AND como operador booleano.

Como critério de elegibilidade, foram inclusos os artigos disponíveis na íntegra, publicados nos últimos cinco anos (2015 - 2020), em português e que possuíam relação direta com a temática abordada, essas informações foram obtidas através dos filtros utilizados e da leitura do artigo completo para posterior decisão de inclusão ou exclusão. Portanto, não foram inseridos os trabalhos em estrutura de monografias, dissertações e teses, textos em outros idiomas, sem conteúdo completo, artigos pagos, relatos de caso e experiência, entre outros estudos considerados inelegíveis.

Dos artigos inclusos, foi elaborado uma avaliação das informações contidas para responder às questões norteadoras deste estudo e uma síntese com exposição e discussão dos resultados que constituiu a revisão integrativa.

\section{RESULTADOS}

Foram encontrados o total de 47 artigos e selecionados 16 para compor este estudo, sendo que 10 (62,5\%) estão relacionados aos CAPS, com estudos que enfatizam as atividades assistenciais e terapêuticas, e seis $(37,5 \%)$ estão ligadas ao SRT, ressaltando a autonomia do indivíduo. A base de dados com maior número de artigos encontrados e selecionados foi o BDENF (56,2\%). Está representado a quantidade de artigos encontrados após utilizar as palavras-chave e os filtros texto completo, idioma e ano de publicação (Quadro 1).

Quanto à peridiocidade que registraram o maior número de publicações inseridas, destacam-se os anos de 2017, com seis artigos incluídos (37,5\%) e o ano de 2016 (31,2\%), com cinco trabalhos publicados. Não foi observado a prevalência de nenhuma revista neste estudo e não houve artigos que respondessem à pergunta norteadora deste trabalho publicados no ano de 2020.

Em relação aos tipos de estudo encontrados para compor esta revisão, na sua grande maioria do tipo descritivo $(43,75 \%)$, mas também constituído de artigos observacionais $(18,75 \%)$, documentais $(6,25 \%)$ e exploratórios (12,5\%). Com relação ao tipo de abordagem, os do tipo qualitativo chegaram a compor $62,5 \%$ e, os de abordagem quantitativa apenas a $12,5 \%$.

Quadro 1 - Busca de artigos em bases de dados com o total de publicações encontradas, selecionadas e excluídas.

\begin{tabular}{|c|c|c|c|c|}
\hline Bases de dados & Palavras-chave (DeCS) & $\begin{array}{c}\text { Publicações } \\
\text { encontradas }\end{array}$ & $\begin{array}{c}\text { Publicações } \\
\text { incluídas }\end{array}$ & $\begin{array}{c}\text { Publicações } \\
\text { excluídas }\end{array}$ \\
\hline LILACS & $\begin{array}{c}\text { Saúde mental, CAPS, } \\
\text { Enfermagem. }\end{array}$ & 15 & 7 & 8 \\
\hline BDENF & $\begin{array}{c}\text { Saúde mental, } \\
\text { Enfermagem. }\end{array}$ & 32 & 9 & 24 \\
\hline
\end{tabular}

Fonte: Santos TMP, et al., 2021.

Realizando um levantamento sobre os anos de publicação dos manuscritos, percebe-se a predominância do ano de 2016, com cerca de 31,25\% (5 publicações), seguido do ano de 2017, com 37,5\% (6 publicações); o ano de 2018 correspondeu apenas a 6,25\% (1) das publicações e, 2019, com apenas 4 publicações (25\%).

Das referências excluídas, os principais motivos foram, conteúdo incompleto, artigos fora da temática proposta e teses de doutorado. Dos artigos extraídos das bases de dados elaborou-se uma tabulação que contém informações baseadas na procedência do trabalho onde estão apresentadas no quadro a seguir (Quadro 2). 
Quadro 2 - Artigos inclusos na revisão integrativa contendo o título, autores, periódicos e as principais considerações temáticas.

\begin{tabular}{|c|c|c|c|c|}
\hline № & $\begin{array}{l}\text { Bases } \\
\text { de } \\
\text { Dados }\end{array}$ & Autor/Ano & Tipo de Estudo & Conclusões \\
\hline 1 & LILACS & Silva AS, et al. (2019) & $\begin{array}{l}\text { Pesquisa descritiva exploratória, qualitativa, realizada em seis SRT no } \\
\text { município do Oeste Paulista. Foram entrevistados } 12 \text { cuidadores, que } \\
\text { contaram como foi a experiência de iniciar as atividades assistências no } \\
\text { SRT; quais são as fragilidades e as potencialidades que envolvem as } \\
\text { atividades assistências. }\end{array}$ & $\begin{array}{l}\text { Identificou dificuldades na reintegração social dos portadores de } \\
\text { transtornos mentais, sendo a mais frequente a dificuldade em } \\
\text { comunicar-se e estabelecer vínculo com os moradores e os } \\
\text { profissionais da residência terapêutica. }\end{array}$ \\
\hline 2 & BDENF & $\begin{array}{l}\text { Nobrega MP e Veiga TF } \\
\text { (2017) }\end{array}$ & $\begin{array}{l}\text { Pesquisa qualitativa, realizada com treze moradores de duas Residências } \\
\text { Terapêuticas, na cidade de São Bernardo do Campo, São Paulo, cuja } \\
\text { finalidade foi compreender o exercício da cidadania. }\end{array}$ & $\begin{array}{l}\text { Os moradores da residência terapêutica relatam satisfação para ter } \\
\text { uma vida mais independente, porém enfrentam desafios para } \\
\text { empoderar-se e fazer escolhas devido suas capacidades cognitivas. }\end{array}$ \\
\hline 3 & LILACS & Maluf RG, et al. (2017) & $\begin{array}{l}\text { Pesquisa observacional e descritiva realizada com } 154 \text { pacientes } \\
\text { psiquiátricos de } 25 \text { unidades de SRT na cidade de Barcelona-MG, onde } \\
\text { abrigavam adultos com transtornos psíquicos graves e persistentes sem } \\
\text { vínculo familiar que relataram o grau de satisfação dos pacientes referente } \\
\text { a competência dos cuidadores a compreensão dos seus problemas e } \\
\text { avaliação do SRT. }\end{array}$ & $\begin{array}{l}\text { Os pacientes relataram alto grau de satifação com o serviço prestado } \\
\text { independente das condições psíquicas, também foi observado que a } \\
\text { maior satisfação foi descrita por usuários que realizavam tratamento } \\
\text { medicamentoso e atividades extras como terapia e hidroginástica. }\end{array}$ \\
\hline 4 & LILACS & $\begin{array}{l}\text { Silva DAB e Vincentin } \\
\text { MCG (2017) }\end{array}$ & $\begin{array}{l}\text { Estudo de observação do cotidiano e atividades externas da RT tais como } \\
\text { passeios e visitas no CAPS. Foi realizado no território da Zona Norte de São } \\
\text { Paulo onde contava com oito moradores adultos e oito profissionais. }\end{array}$ & $\begin{array}{l}\text { Tem efeitos positivos nos moradores de residência terapêutica, na } \\
\text { reconstrução dos sentidos/valores de reconstrução de tempo e } \\
\text { responsabilização. }\end{array}$ \\
\hline 5 & LILACS & $\begin{array}{l}\text { Candido MCCM } \\
\text { Lavrador MCC (2017) }\end{array}$ & $\begin{array}{l}\text { Pesquisa realizada com moradores de um SRT, antigos internos de um } \\
\text { hospital psiquiátrico, na região metropolitana de Vitória-ES. Foram } \\
\text { coletados dados feitos em cadernos de campo cujo a finalidade é saber se } \\
\text { os antigos internos são capazes de voltar a fortalecer valores e situações da } \\
\text { vida cotidiana. }\end{array}$ & $\begin{array}{l}\text { Os participantes apresentaram dificuldades frequentes em } \\
\text { ressocializar devido a internações prolongadas e estadias em } \\
\text { manicômios, portanto foi possível concluir que a adaptação dessas } \\
\text { pessoas ao convívio social é um desafio contínuo. }\end{array}$ \\
\hline 6 & LILACS & Souza AC, et al. (2019) & $\begin{array}{l}\text { Trata-se de uma pesquisa exploratória, transversal e qualitativa realizada } \\
\text { em } 13 \text { CAPS da cidade do Rio de Janeiro e teve como finalidade estudar a } \\
\text { realidade da implantação das ações de saúde mental na atenção básica de } \\
\text { saúde e mostrar a integralidade no atendimento. }\end{array}$ & $\begin{array}{l}\text { Ampliou o acesso dos usuários aos cuidados que necessitam com o } \\
\text { atendimento para doenças frequentes, promoção e prevenção a } \\
\text { saúde na atenção primária. Relatou a importância do cuidado integral } \\
\text { a esses pacientes identificando outras patologias distintas. }\end{array}$ \\
\hline 7 & BDENF & Oliveira EM, et al. (2019) & $\begin{array}{l}\text { Um estudo documental, retrospectivo com abordagem quantitativa, foi } \\
\text { realizado no CAPS ad de um município de referência do estado do Ceará. } \\
\text { Foi realizado o perfil sociodemográfico através da leitura dos prontuários } \\
\text { dos usuários e observou a relevância da enfermagem nesse centro } \\
\text { especializado. }\end{array}$ & $\begin{array}{l}\text { Observou o predomínio de homens com o ensino fundamental } \\
\text { incompleto e enfatizou a relevância do atendimento da equipe de } \\
\text { enfermagem para minimizar as internações já que desempenham } \\
\text { papel fundamental nesses centros. }\end{array}$ \\
\hline 8 & BDENF & $\begin{array}{l}\text { Silva JVS e Brandão TM } \\
\text { (2019) }\end{array}$ & $\begin{array}{l}\text { Pesquisa descritiva de abordagem quantitativa realizada em } 5 \text { CAPS de } \\
\text { Maceió-AL. Foram entrevistados profissionais de enfermagem afim de } \\
\text { identificar as suas atribuições em um CAPS, as funções assistenciais e as } \\
\text { relacionadas a reintegração dos pacientes baseados no cotidiano e relato } \\
\text { dos profissionais. }\end{array}$ & $\begin{array}{l}\text { Percebeu-se que os enfermeiros desempenham diversas funções } \\
\text { como supervisão, curativos simples, consultas participação em } \\
\text { grupos terapêuticos, visitas domiciliares e projeto terapêutico } \\
\text { singular que é afetado devido à falta de recurso financeiro. }\end{array}$ \\
\hline
\end{tabular}




\begin{tabular}{|c|c|c|c|c|}
\hline № & $\begin{array}{l}\text { Bases } \\
\text { de } \\
\text { Dados }\end{array}$ & Autor/Ano & Tipo de Estudo & Conclusões \\
\hline 9 & BDENF & Willrich JQ, et al. (2018) & $\begin{array}{l}\text { Um estudo descritivo de abordagem qualitativa, realizada em } 2016 \text { em um } \\
\text { CAPS II em uma cidade do Rio Grande do Sul. Foi observado a capacidade } \\
\text { de interação dos pacientes nas oficinas terapêuticas propostas por } \\
\text { profissionais de um centro psicossocial. }\end{array}$ & $\begin{array}{l}\text { Observou-se que as oficinas terapêuticas são benéficas para saúde } \\
\text { e promoveram a inclusão e criação de vínculo, resultando em } \\
\text { demonstração de afeto com os profissionais. }\end{array}$ \\
\hline 10 & LILACS & Guedes AC, et al. (2017) & $\begin{array}{l}\text { Pesquisa qualitativa desenvolvida no CAPS II de um município de grande } \\
\text { porte em Santa Catarina em 2014. Observou o processo de alta dos } \\
\text { usuários com enfoque na avaliação dos usuários, familiares e profissionais } \\
\text { do CAPS, para compreender a utilização da alta enquanto transferência de } \\
\text { cuidados. }\end{array}$ & $\begin{array}{l}\text { Para os profissionais, o termo alta não se aplica, e sim a transferência } \\
\text { de cuidados, que o indivíduo estável frequente outras modalidades } \\
\text { de atenção a saúde como a UBS. O vínculo com o CAPS tem que } \\
\text { existir, não para aprisionar o indivíduo e sim realizar um } \\
\text { acompanhamento adequado. }\end{array}$ \\
\hline 11 & BDENF & Silveira MPR, et al. (2017) & $\begin{array}{l}\text { Estudo exploratório e qualitativo, realizado com } 21 \text { participantes, sendo } 11 \\
\text { familiares e } 10 \text { profissionais do CAPS ad. Foram coletados dados através } \\
\text { de entrevista exploratória para avaliar o grau de autonomia e contribuição } \\
\text { do tratamento na reintegraçãa social. }\end{array}$ & $\begin{array}{l}\text { Os profissionias entrevistados descrevem que a abstinência total do } \\
\text { uso de drogas é um proceso demorado e que a redução de danos } \\
\text { que consiste em diminuir as doses utilizadas sirva de incentive para } \\
\text { continuidade no tratamento paar posterior reintegração social, o que } \\
\text { pelo lado familiar difere visto que acreditam que o ideal é se manter } \\
\text { em abstinência total. }\end{array}$ \\
\hline 12 & BDENF & Baptista J, et al. (2016) & $\begin{array}{l}\text { Estudo qualitativo realizado em um CAPS III do Rio de Janeiro em } 2013 . \\
\text { Foram coletados dados através de observação para avaliar a capacidade } \\
\text { dos usuários de se vincular ao serviço. }\end{array}$ & $\begin{array}{l}\text { O ambiente terapêutico do CAPS favorece a proposta de cuidado, } \\
\text { ajudando usuários se vincular ao serviço, construir projeto de vida, } \\
\text { melhorar sua sociabilidade, se fortalecer como cidadão, melhorar o } \\
\text { nível de saúde. }\end{array}$ \\
\hline 13 & BDENF & Brandão TM, et al. (2016) & $\begin{array}{l}\text { Pesquisa de caráter descritivo, qualitativo, onde teve como participantes } \\
\text { sete enfermeiros do CAPS de uma capital do Nordeste. }\end{array}$ & $\begin{array}{l}\text { A práxis do enfermeiro dentro do CAPS deve-se principalmente em } \\
\text { grupos terapêuticos, educação em saúde, visita domiciliar, } \\
\text { administração de medicamentos e acolhimento. }\end{array}$ \\
\hline 14 & LILACS & Farias ID, et al. (2016) & $\begin{array}{l}\text { Estudo de abordagem qualitativa realizada em Pelotas-RS, em um CAPS II. } \\
\text { Foram coletados dados através de entrevista com } 24 \text { profissionais com o } \\
\text { objetivo de identificar os profissionais que atuam nas oficinas terapêuticas e } \\
\text { suas contribuições. }\end{array}$ & $\begin{array}{l}\text { Mostrou que as oficinas terapêuticas foram identificadas como } \\
\text { espaços que propiciam os pacientes de saúde mental meios de } \\
\text { buscar suas potencialidades valorizando os aspectos saudáveis da } \\
\text { vida, permitindo a expressão através da arte. }\end{array}$ \\
\hline 15 & BDENF & Pires LFB, et al. (2016) & $\begin{array}{l}\text { Um estudo descritivo realizado em um CAPS do município de grande porte } \\
\text { do Sudeste Brasileiro com } 825 \text { pacientes dependentes químicos de álcool } \\
\text { e/ou drogas em tratamento. }\end{array}$ & $\begin{array}{l}\text { Observou que o atendimento ao dependente químico não foi } \\
\text { realizado de forma integrada na maioria dos casos e alerta sobre o } \\
\text { fortalecimento das ações conjuntas para a recuperação dos } \\
\text { pacientes. }\end{array}$ \\
\hline 16 & LILACS & Silva AB, et al. (2016) & $\begin{array}{l}\text { Um estudo qualitativo e descritivo realizado em um CAPS ad de uma cidade } \\
\text { da região metropolitana de Porto Alegre. Dados foram coletados com } 8 \\
\text { profissionais através de uma entrevista por território no cuidado ao usuário } \\
\text { de crack. }\end{array}$ & $\begin{array}{l}\text { A equipe itinerante que realiza visitas domicilires e presta assistência } \\
\text { especializada fora do CAPS é uma estratégia eficaz de acordo com } \\
\text { os profissionais pois permite que a equipe vá de encontro ao paciente } \\
\text { quebrando barreiras geográficas e incentivando a conclusão do } \\
\text { tratamento. }\end{array}$ \\
\hline
\end{tabular}

Fonte: Santos TMP, et al., 2021. 


\section{DISCUSSÃO}

Nas publicações relacionadas à enfermagem e saúde mental nos últimos cinco anos, nota-se o protagonismo dos CAPS ad e CAPS III, entretanto, para os CAPS i que atendem o público infanto juvenil não foram localizadas nenhuma publicação, dessa forma foi possível constatar um déficit de interesse em pesquisar as limitações do cotidiano dessas populações. Quanto as demais modalidades de assistência, como os SRT, foram encontradas poucos estudos quando comparado aos artigos sobre CAPS. Atualmente já são conhecidas alternativas de atendimento para pacientes com distúrbios mentais, como hospital dia e projeto terapêutico singular que precisam de enfoque, mas não recebem incentivo para manter sua funcionabilidade e, consequentemente, não são temáticas muito abordadas em artigos científicos.

\section{Contribuição do Serviço de Residência Terapêutica no processo de Reintegração Social}

Dos trabalhos publicados acerca da eficácia do Serviço de Residência Terapêutica (SRT), Nobrega MPSS, et al. (2017) descrevem os principais obstáculos dos usuários, dentre as queixas, a mais relevante foi a percepção da falta de autonomia relacionada às condições psíquicas instáveis, o que ocasiona a falta de empoderamento individual. Esses dados corroboram com o estudo de Canido MCCM, et al. (2017) cujo resultado predominante foi a dificuldade dos moradores do SRT em se adaptar as mudanças após a reforma psiquiátrica, o que prejudica a relação interpessoal. Os cuidadores desses serviços de longa permanência possuem função primordial no estímulo da comunicação e verbalização dos internos com pessoas sem nenhum transtorno mental, por isso, pode-se considerar essa conversação como uma avaliação cognitiva afim de identificar a presença ou ausência de falas desconexas além de contribuir com a ressocialização.

Quanto ao grau de satisfação do serviço prestado, Silva DAB, et al. (2017) destacam a importância da SRT para a construção de valores e criação de uma rotina. Semelhantemente, Maluf RG, et al. (2017) que avaliaram os benefícios da desinstitucionalização psiquiátrica, tem como resultado principal a satisfação da maioria dos residentes do SRT que consideram esta modalidade de atendimento psiquiátrico efetiva, sendo assim, em ambos os estudos concluíram que há ressocialização e melhora na qualidade de vida, demonstrando resultados positivos e satisfação dos usuários desse serviço.

A atuação profissional nas residências terapêuticas é uma abordagem de difícil publicação, isso evidenciase pelo fato de ter sido encontrado e incluído apenas um trabalho escrito por Silva AST, et al. (2019). Nesta pesquisa foi demonstrado a sobrecarga profissional dos cuidadores responsáveis pela manutenção desses lares, pois além de fornecer um ambiente limpo e alimentação, eles devem manter contato com os moradores, o que dificilmente ocorre, resultando em falhas no processo de comunicação. Como os pacientes que utilizam o SRT em sua maioria são egressos de manicômios, estes podem apresentar incapacidade de realizar a higiene pessoal, comportamentos agressivos, falas desconexas, entre outros, por estes motivos, a interação cuidador e morador possui tantos obstáculos.

\section{Assistência de Enfermagem em Centros de Atenção Psicossocial}

A saúde mental com ênfase intra-hospitalar e reclusiva vem sendo desconstruída desde a reforma psiquiátrica, em um estudo feito por Souza AC, et al. (2019), enfermeiros que relataram a relevância do atendimento na Atenção Básica e propuseram a ampliação da assistência psiquiátrica em CAPS do Rio de Janeiro, o que resultou na mudança holística dos gestores das unidades. A proposta central foi tornar o atendimento integral para pacientes que possuem distúrbios psíquicos, facilitando o acesso à prevenção e promoção à saúde. Implementar essa estratégia de cuidado amplia a quantidade de serviços fornecidos, sendo possível retirar usuários que permanecem em setor terciário especializado, sem necessidade para que possam realizar o acompanhamento extra-hospitalar na atenção primária, contribuindo no processo de reintegração social.

As contribuições da assistência de enfermagem frente a um CAPS foram publicadas por Silva JVS, et al. (2019) que observaram a influência positiva no convívio dos pacientes e como o enfermeiro atua nesses centros. Relacionando a assistência do enfermeiro com o processo de alta dos pacientes, Guedes AC, et al. (2017) destacam a participação da enfermagem que visa a reabilitação psicossocial e contribui no processo de alta desses pacientes. 
O CAPS ad atende os casos de pacientes com transtornos relacionados ao uso abusivo de álcool e drogas, nesta revisão foram encontrados mais artigos publicados relacionados a essa modalidade, o que evidencia 0 interesse por essa linha de pesquisa. O perfil epidemiológico presente no artigo de Oliveira EN, et al. (2019) destacam a prevalência do sexo masculino nos casos de internação em CAPS ad, e de forma complementar, Pires LFB, et al. (2016) identificam que quando relacionado ao fumo, as mulheres mantêm o maior número de internações, entretanto quando comparado a utilização de álcool, os homens. No artigo de Pires LFB, et al. (2016), os autores concluem a importância do cuidado multiprofissional sendo que nas categorias mais relevantes do atendimento, encontram-se os técnicos de enfermagem e enfermeiros que desempenham papel primordial com assistência ao paciente usuário de drogas.

A capacidade cognitiva de um indivíduo com dependência química tende a ser comprometida, entretanto através do acompanhamento e tratamento ela pode ser reestabelecida, no olhar profissional e familiar no estudo de Silva JVS, et al. (2019), os parentes de pacientes internados em CAPS ad compreendem a importância da ressocialização, entretanto, relataram diversos obstáculos, como a dificuldade de lidar com a abstinência, manter vínculos afetivos e facilidade em comprar e utilizar drogas o que leva a sucessivas internações. No olhar profissional o relato assemelha-se ao ponto de vista familiar e ambos acreditam que os pacientes podem retornar a um estado cognitivo padrão, apto para realizar suas rotinas de forma independente. Por isso, Silva AB, et al. (2016) propõe que haja maior atenção voltada para a parte social e cultural dos indivíduos com o intuito de investir em outras habilidades do indivíduo das quais ele possa se dedicar.

\section{Principais atribuições dos profissionais de enfermagem na saúde mental}

A prática do processo de cuidados da enfermagem é amplamente bem distribuída e faz-se necessária em todos os níveis de assistência, o artigo de Brandão TM, et al. (2016) discorre acerca de algumas das atribuições do profissional enfermeiro especializado em saúde mental, sendo a visita domiciliar e a administração de medicamentos as principais atividades assistenciais realizadas, o trabalho de Silva JVS, et al. (2019) complementa identificando todas as atividades assistenciais prestadas dentro de um CAPS pela equipe de enfermagem e o profissional enfermeiro.

As atuações dos enfermeiros citados nesse artigo incluem supervisão e gerência da equipe de enfermagem, realização de curativos simples, Sistematização da Assistência de Enfermagem (SAE) e participação no projeto terapêutico singular. Este projeto pode ser utilizado como ferramenta para determinar o grau de comprometimento psíquico dos pacientes, entretanto, não são disponibilizados os recursos para sua execução frequente, a falta de verba é um dos principais obstáculos na busca de uma terapia acolhedora e integral para os usuários do CAPS (BRANDÃO TM, et al., 2016).

\section{Ações e estratégias que podem contribuir para o processo de reintegração social de pacientes psíquicos}

A enfermagem é protagonista na Atenção Básica em Saúde (ABS), sendo assim, inserida em diversas esferas de assistência, incluindo a saúde mental. Estudos mostram que a principal barreira para um atendimento efetivo e que dificultam a implementação de estratégias ou ações é a falta de comunicação. Como o processo de reintegração social depende diretamente dos CAPS e da participação dos assistidos para sua reabilitação e prevenção, logo os ruídos e falhas de comunicação afetam a integralidade do atendimento (CECILIO LCO e REIS AAC, 2018).

Dentre as atividades lúdicas e terapêuticas realizadas por enfermeiros capazes de avaliar o estado cognitivo dos pacientes, encontram-se as oficinas de música, rodas de conversa e arteterapia. A arteterapia utilizada em um CAPS por Willich JQ, et al. (2018) serviu para estabelecer um vínculo afetivo entre os profissionais e pacientes além de demonstrarem interesse pela atividade realiza, por isso, nesse estudo concluíram que a arte influencia no aprimoramento de funções cognitivas além de ser benéfica para a saúde, esses dados ratificam com os apresentados por Batispta J, et al. (2016) e Farias I, et al. (2017) que observaram o processo de contribuição das oficinas terapêuticas na sociabilidade eficaz dos indivíduos valorizando sua singularidade através da expressão artística. Estes autores também destacam a arte como forma de distração, estes momentos lúdicos permitem interações sociais de forma descontraída e espontânea, nos quais conseguem criar laços afetivos por proximidade com quem mais se identificam. 
No estudo de Estevam AS, et al. (2020), oficinas de enfermagem são vistas como estratégias importantes para o desenvolvimento de ideias e levantamento de críticas que favoreçam uma discussão sobre a importância da reintegração social, contribuindo para a atuação com competência. Os mesmos autores corroboram com o fato de que as Instituições de Ensino Superior (IES) necessitam retomar a abordagem do tema para que novas metodologias possam ser atribuídas.

\section{CONSIDERAÇÕES FINAIS}

O processo de reintegração social é complexo e adapta-se as diferentes condições psíquicas dos pacientes variando os resultados entre os estudos. Visando o atendimento extra hospitalar com acesso integral, os CAPS e os serviços de residência terapêutica correspondem a estratégias em saúde mental que mais contribuem para a ressocialização dos indivíduos, entretanto, a falta de recurso e a sobrecarga profissional, retardam o resultado esperado. O profissional enfermeiro e a sua equipe realizam atividades assistências como terapias comportamentais, visitas domiciliares, consultas de enfermagem e participação no projeto terapêutico singular. Deste modo, foi possível compreender que há relevância da assistência de enfermagem no processo de reintegração social, enfatizando a autonomia e singularidade de cada indivíduo.

\section{REFERÊNCIAS}

1. BAPTISTA J, et al. A importância do ambiente terapêutico no cuidado em um Centro de Atenção Psicossocial. Revista de Enfermagem UFPE Online, 2016; (6)10: 4711-4719.

2. BRANDÃO TM, et al. A práxis do enfermeiro na atenção psicossocial: Vulnerabilidades e potencialidades presentes. Revista de Enfermagem UFPE Online, 2016; (6)10: 4766-4777.

3. BRASIL. Diário Oficial da União. Decreto nำ 1.132, de 22 de dezembro de 1903: reorganiza a assistencia a alienados. 1903. Acesso em: 15 abr. 2020.

4. BRASIL. Ministério da Saúde. Nota técnica n 11/2019 - CGMAD/DAPES/SAS/MS: Esclarecimentos sobre as mudanças na Política Nacional de Saúde Mental e nas Diretrizes da Política Nacional sobre Drogas. Brasília, 2019. $32 \mathrm{p}$.

5. BRASIL. Ministério da Saúde. Os Centros de Atenção Psicossocial. 2004. Brasília. Acesso em: 15 abr. 2020.

6. BRASIL. Ministério da Saúde. Reforma psiquiátrica e política de saúde mental no Brasil. 2005. Brasília. Acesso em: 15 abr. 2020.

7. BRASIL. Setor de Embaixadas do Norte. Folha informativa - Transtornos mentais: determinantes sociais e riscos para a saúde, doenças crônicas não transmissíveis e saúde mental. Determinantes Sociais e Riscos para a Saúde, Doenças Crônicas não transmissíveis e Saúde Mental. 2018. OMS Brasil. Acesso em: 15 abr. 2020.

8. CABRAL SB, DAROSCI M. A Trajetória das políticas de saúde mental no Brasil: uma análise a partir do ângulo normativo (1903-2019). Universidade Federal de Santa Catarina, 2019; 1-13.

9. CANDIDO MCM, LAVRADOR MCC. Intermitências infames: loucura e alteridade na cidade aberta. Revista Polis e Psique, 2017; 2(7): 46-62.

10. CECILIO LCO, REIS AAC. Apontamentos sobre os desafios (ainda) atuais da atenção básica à saúde. Cadernos de Saúde Pública [online]. 2018; (34)8: e00056917.

11. CONSELHO FEDERAL DE ENFERMAGEM (COFEN). Resolução Cofen 599/2018. Aprova norma técnica para atuação da equipe de enfermagem em saúde mental e psiquiatria. Brasília (DF): Cofen, 2018.

12. EMERIM MF, SOUZA M. Ninguém esquece uma coisa dessas": problematizações sobre parricídio e hospitais de custódia: problematizações sobre parricídio e hospitais de custódia. Psicologia \& Sociedade, 2016, 28(1): 171-180.

13. ESTEVAM AS, et al. A enfermagem em saúde mental pós-reforma psiquiátrica. Revista Eletrônica Acervo Saúde. 2020; 45: e2631.

14. FARIAS I, et al. Oficina terapêutica como expressão da subjetividade. Revista Eletrônica Saúde Mental Álcool e Drogas, 2016; 12(3): 147-153.

15. GUEDES AC, et al. Transferência de cuidados: processo de alta dos usuários de um centro de atenção psicossocial. Revista Eletrônica de Enfermagem, 2017; 9(1): 1-9.

16. KINKER FS. Um hospício em crise: imagens de uma experiência de desinstitucionalização. Interface - Comunicação, Saúde, Educação, 2017; 21(60): 189-198.

17. MALUF RG, et al. Psychiatric patients' satisfaction in the therapeutic residence services: a positive experience of psychiatric deinstitutionalization. Estudos de Psicologia: Periódicos eletrônicos em psicologia, 2017; 22(3): 275-284.

18. MARTINS GCS, et al. Teaching undergraduate nursing in mental health as allied to the consolidation of the Psychiatric Reform movement. Escola Anna Nery, 2018; 22(4): 1-8. 
19. MEDEIROS DAA, et al. Avaliação das limitações do comportamento social dos moradores dos serviços residências terapêuticas de um pequeno município do estado do Rio de Janeiro. Cadernos Saúde Coletiva, 2018; 26(3):278-284.

20. NOBREGA MP, VEIGA TF. O significado de morar em um serviço residencial terapêutico. Revista de Enfermagem do Centro-oeste Mineiro, 2017; 7: 1-8.

21. OLIVEIRA EN, et al. O cuidado multiprofissional na prevenção de internações relacionadas ao uso de crack. Rev. de Pesquisa: Cuidado é fundamental, 2019; 11(5): 293-1299.

22. PINHEIRO CW. Teoria das relações interpessoais: reflexões acerca da função terapêutica do enfermeiro em saúde mental. Enfermagem em Foco, 2019; 3(10): 64-69.

23. PIRES LFB, et al. Estratégia saúde da família e assistência ao dependente químico: ações conjuntas ou isoladas?. Revista Eletrônica de Enfermagem, 2016; 18: 1-11.

24. SILVA $A B$, et al. $O$ cuidado ao usuário de crestratégias e práticas de trabalho no território. Revista Gaúcha de Enfermagem, 2016; 37: 1-7.

25. SILVA AST, et al. Ser cuidador em um serviço residencial terapêutico: fragilidades e potencialidades na prática assistencial. J. nurs. Health, 2019; 9: 109.

26. SILVA DAB, VINCENTIM MCG. Cotidiano de uma residência terapêutica e a produção de subjetividade. Distúrbios da Comunicação: Distúrb. comum, 2017; 29(2):196-206.

27. SILVA JVS, BRANDÃO TM. A enfermagem dos centros de atenção psicossocial de uma capital do nordeste do brasil. Revista de Enfermagem e Atenção à Saúde, 2019; 8(1): 27-38.

28. SOUZA AC, et al. Inclusion of mental health in primary health care: care strategy in the territory. Revista Brasileira de Enfermagem, 2019; 72(6): 1677-1682.

29. SOUZA MT, et al. Integrative review: What is it? How to do it?. Einstein (São Paulo), 2010; 8(1):102-106.

30. SILVEIRA MPR, et al. Autonomia e reinserção social: percepção de familiares e profissionais que trabalham com redução de danos / autonomy and social reintegration. Ciência, Cuidado e Saúde, 2017; 16(3): 1-7.

31. WILLRICH JQ, et al. Atividades de arteterapia na reabilitação de usuários da atenção psicossocial. Revista de Enfermagem e Atenção à Saúde, 2018; 3(7): 50-62. 